\title{
Nutritional Biomarker Analyses for Hypertension-Related Risk Assessment and Control
}

\author{
Mari Mori, Hideki Mori, Yukio Yamori \\ Institute for World Health Development, Mukogawa Women's University, Nishinomiya, Japan \\ Email: yamori@cardiacstudy.com
}

Received 5 April 2014; revised 9 May 2014; accepted 16 May 2014

Copyright (C) 2014 by authors and Scientific Research Publishing Inc.

This work is licensed under the Creative Commons Attribution International License (CC BY).

http://creativecommons.org/licenses/by/4.0/

(c) (i) Open Access

\section{Abstract}

A simple method to collect 24-hour urine (24U) by sampling $2.5 \%$ of the voided urine each time was developed for analyzing the biomarkers of the intakes of salt $(\mathrm{NaCl})$, vegetables and grains $(\mathrm{K}$, Mg), soys (isoflavones), sea-foods (taurine), protein (urea nitrogen) etc. This method was applied to WHO-coordinated CARDIAC (Cardiovascular Diseases and Alimentary Comparison) study covering 61 populations in 25 countries, and revealed significant positive associations of $\mathrm{Na}$ or $\mathrm{Na} / \mathrm{K}$ with blood pressure (BP) and stroke mortality and significant inverse associations of isoflavones or taurine with coronary heart disease (CHD) mortality, indicating the adverse effect of salt intake on BP and stroke and beneficial effect of soy or sea food intakes on CHD. Furthermore, higher urinary taurine and/or Mg excretions were associated with significantly lower risks of metabolic syndromes such as obesity, hypertension and hypercholestelomia. $24 \mathrm{U}$ collections were useful to prove nutritional improvement by intervention studies for observing soy intake effect on BP and atherogenic index in Japanese immigrants living in Brazil, and in Australian Aboriginals, for observing taurine effect on BP in Tibetans and for confirming soy and fish effects on the risks of metabolic syndromes in Japanese businessmen. $24 \mathrm{U}$ collection was utilized for population strategy to reduce salt and to increase soy intake in 5.6 million people of Hyogo Prefecture. A simple method for $24 \mathrm{U}$ collection has enabled us to utilize nutritional biomarkers for hypertension-related disease risk analyses, and to apply objective nutritional intake estimation to intervention studies as well as to population strategy for nutritional improvement, and therefore, the methodology will contribute to hypertension-related disease prevention and global health promotion.

\section{Keywords}

24-Hour Urine, Sodium, Taurine, Isoflavone, Magnesium 


\section{Introduction}

A marked reduction of stroke in the stroke-prone spontaneously hypertensive rats (SHRSP), which were developed as genetic stroke model in Japan [1], confirmed the nutritional influence on cardiovascular diseases (CVD) in the rat models and led us to investigate the association of nutrition with CVD in humans by utilizing nutritional biomarkers. For nutritional biomarker analysis a simple method for 24-hour urine (24U) collection was developed firstly, and secondly the device was successfully applied to worldwide epidemiological survey for analyzing the association between nutritional biomarkers and hypertension-related CVD risks. Thirdly, this method was further applied to nutritional intervention studies and finally to population strategy for health promotion.

\section{Simple Method for 24-Hour Urine (24U) Collection}

Yamori et al. could successfully develop a simple method for collecting 24U samples [2]. By pushing only once the rod of the double bottomed urine cup (Figure 1), 2.5\% of the voided urine can be collected into the under compartment and the rest of the urine is discarded. This simple procedure is repeated for 24-hours to collect a 24U sample precisely. The samples are frozen to ship to an analysis center for measuring $\mathrm{Na}, \mathrm{K}$, taurine, isoflavones and urea nitrogen, for example, as the biomarkers of salt, vegetable, seafood, soybean and total protein intakes.

\section{24U Biomarkers and Hypertension-Related CVD}

This simple method was utilized for investigating the association of nutrition with CVD risks in CARDIAC study covering 61 populations of 25 countries [3]-[6].

CARDIAC study includes populations with food culture good for health and longevity, populations with unfavorable food culture for longevity such as Australian aboriginals, and populations with deteriorating food culture such as Japanese immigrants living in Brazil (Figure 2).

Salt intakes in average in various populations were analyzed by the $24 \mathrm{U}$ collection and the significant associations of daily salt intakes with systolic and diastolic BP (SBP, DBP) were proven (Figure 3) [4]. The lowest intake was noted in Masai people who used no salt at all and showed almost the lowest BP in the world. Since higher BP caused stroke, CARDIAC study proved the age-adjusted mortality rates of stroke were significantly positively related with $24 \mathrm{U}$ salt excretion (Figure 4) [5] [6]. This data indicate the reduction of salt intake less than $6 \mathrm{~g}$ daily should contribute to the great reduction of stroke mortality. Stroke mortality rates were significantly positively related also with $24 \mathrm{U} \mathrm{Na} / \mathrm{K}$ ratios, indicating the importance of the balance of salt intake with $\mathrm{K}$ intake from vegetables and so on in stroke prevention [7].

As for coronary heart disease (CHD) mortality, in addition to the well-known significant positive association with serum cholesterol levels, CARDIAC study first demonstrated the mortality was significantly inversely related with 24U taurine (T) excretion, the reliable biomarker of seafood intake, indicating clearly the beneficial preventive effect of fish consumption on CHD (Figure 5) [5] [8]. T is a simple sulfur amino acid contained rich in seafoods and its beneficial effects such as antihypertensive, hypocholesterolemic and antiatherogenic effects have been proven experimentally in our rat models and also epidemiologically [8] [9]. Fish consumption was positively related with $24 \mathrm{U} \mathrm{T}$ excretions in various populations [8].

Participants in CARDIAC study health examination in the world were divided into 2 groups, greater T excreters excreting $24 \mathrm{U} \mathrm{T}$ over the mean of the world and lesser $\mathrm{T}$ excreters excreting $24 \mathrm{U} \mathrm{T}$ less than the mean. Greater T excreters showed significantly lower BMI, lower SBP and DBP and lower total cholesterol levels than the lesser T excreters [9].

Among various urinary biomarkers, 24U magnesium (Mg) excretion was strongly inversely related with cardiovascular risks [9]. Greater Mg excreters excreting 24U Mg over the mean of the world showed significantly lower BMI, lower SBP and DBP, and lower total cholesterol levels than lesser Mg excreters. Mg is an essential cofactor for ATP-dependent enzymes, so that it is involved in many important biological processes. Then, the total CARDIAC study participants were divided into 4 groups by the means of $24 \mathrm{U} \mathrm{T}$ and $\mathrm{Mg}$ (Figure 6). Greater T and Mg excreters showed significantly lower BMI, lower SBP and DBP, and lower total cholesterol (TC) levels than lesser T and Mg excreters, indicating $\mathrm{T}$ and $\mathrm{Mg}$ intakes might be determinants of cardiovascular risks. 


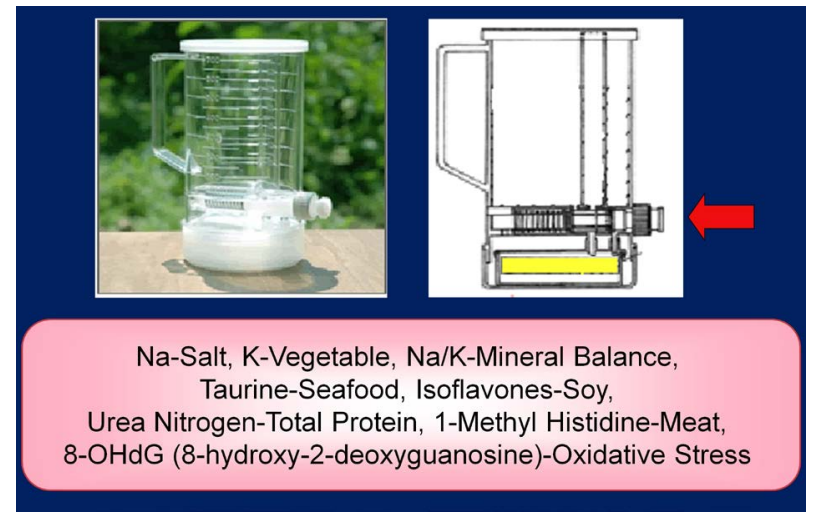

Figure 1. Device for 24-hour urine (24U) collection called aliquot cup or Yamori's cup and analyzed biomarkers for the estimation of nutritional intakes or balance.

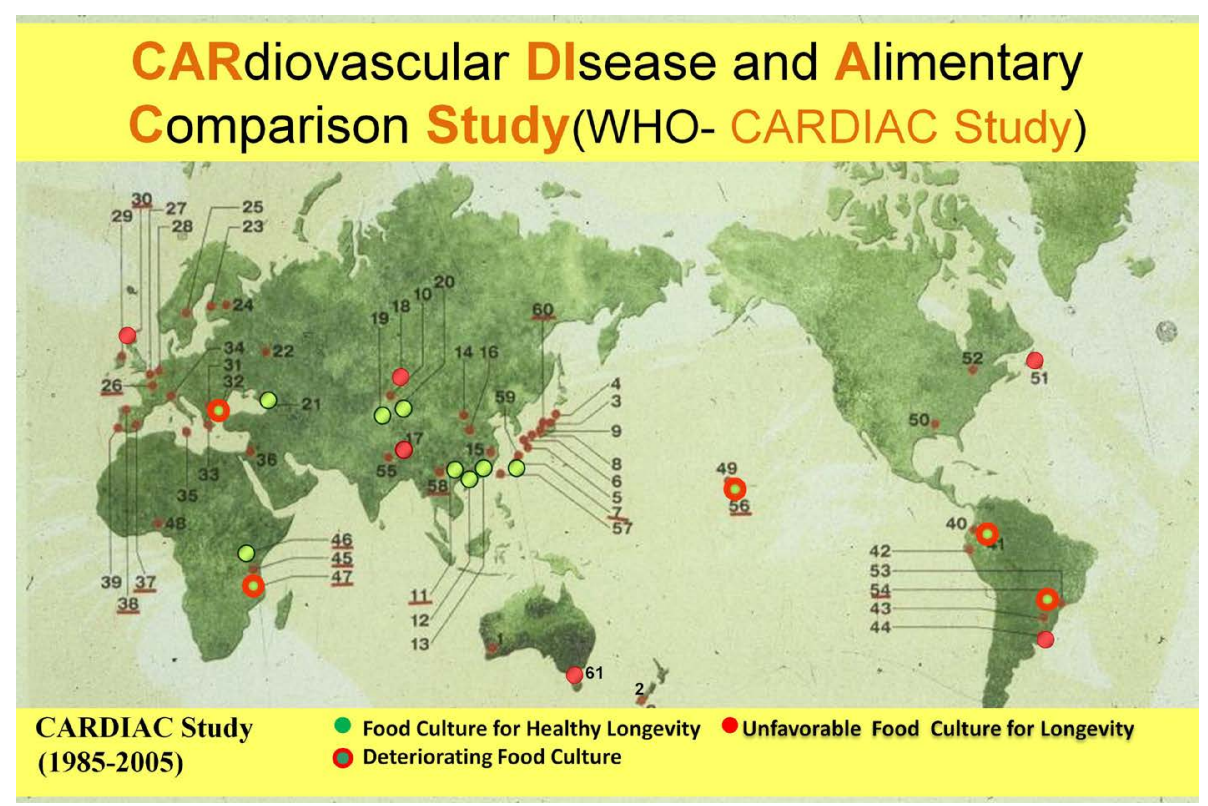

Figure 2. Study sites of cardiovascular disease and alimentary comparison study (WHOCARDIAC study).

Five diet-related factors, total cholesterol, BMI and 24U Na, Mg and T excretions were analyzed in relation to CHD mortality rates by structural equation modeling. TC and BMI were significantly positively associated with CHD, but 24U Mg and T excretions were significantly inversely related with CHD in males and females, indicating $\mathrm{T}$ and Mg could be preventive nutritional factors for CHD [9].

$\mathrm{Mg}$ are rich in nuts, legumes and soy beans containing isoflavones, the structure of which is similar to estrogen. Isoflavones have weak estrogenic effect and are therefore called phytoestrogen. CARDIAC study proved first time in the world the significant inverse association of CHD mortality with $24 \mathrm{U}$ soy isoflavone excretions, indicating the beneficial effect of soy bean intake for the prevention of CHD (Figure 7) [6] [10].

Isoflavones, like estrogen were experimentally proven to accelerate the gene expression of nitro oxide (NO) synthase in vascular endothelial cells, thus to increase NO production. NO dilates blood vessels and has antithrombotic effect and its effect is intensified by antioxidants [10].

\section{24U Biomarker Utilization for Nutritional Intervention}

Since CARDIAC study proved urinary biomarker analyses were useful for CVD risk assessment, nutritional in- 


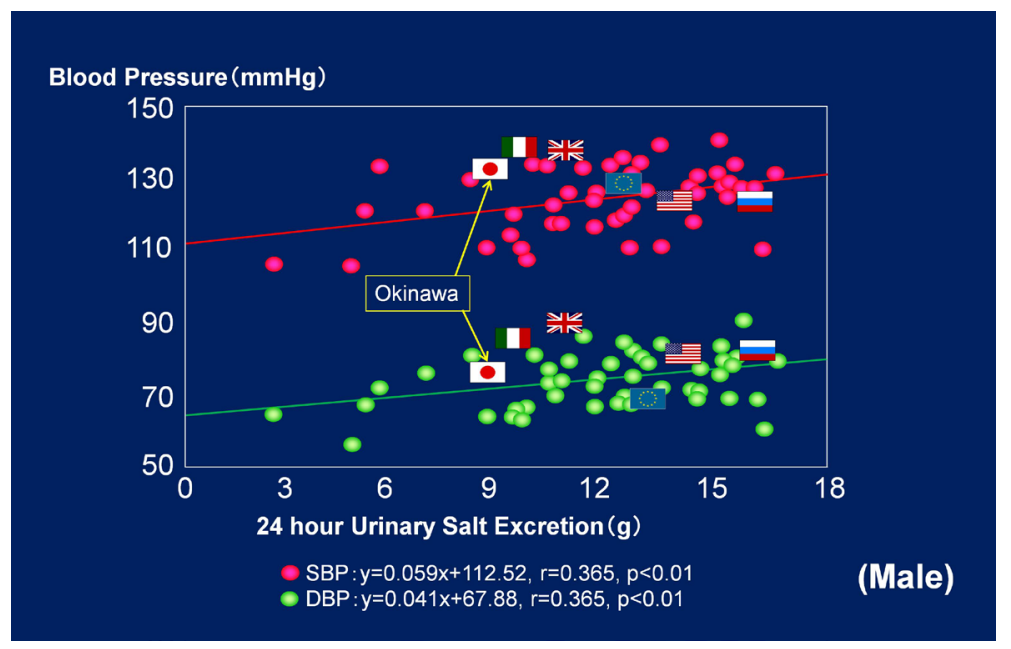

Figure 3. Salt intake estimated from $24 \mathrm{U} \mathrm{NaCl}$ excretion and blood pressure (SBP, DBP).

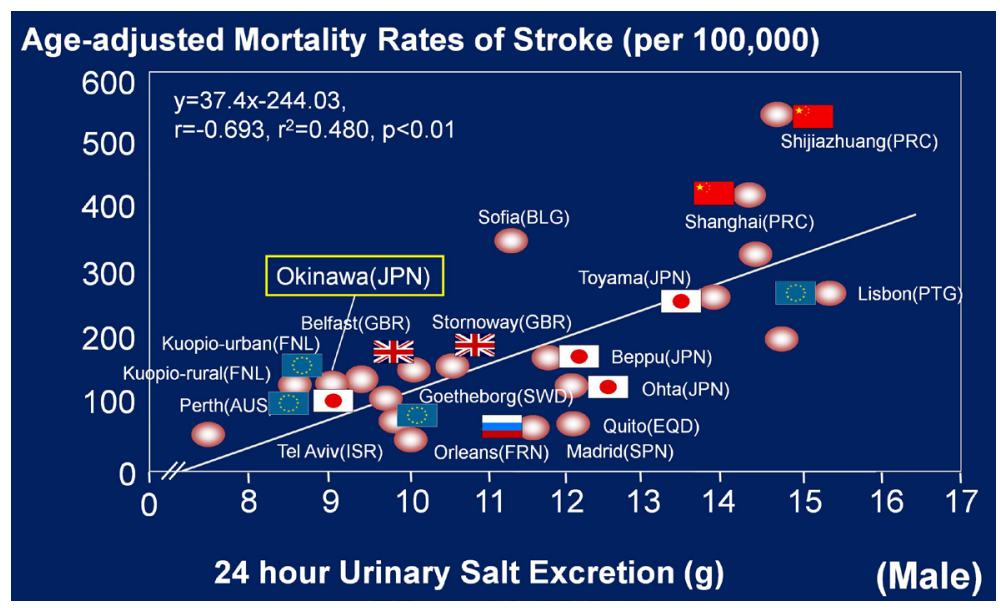

Figure 4. Salt intake estimated from $24 \mathrm{U}$ and age-adjusted mortality rates of stroke.

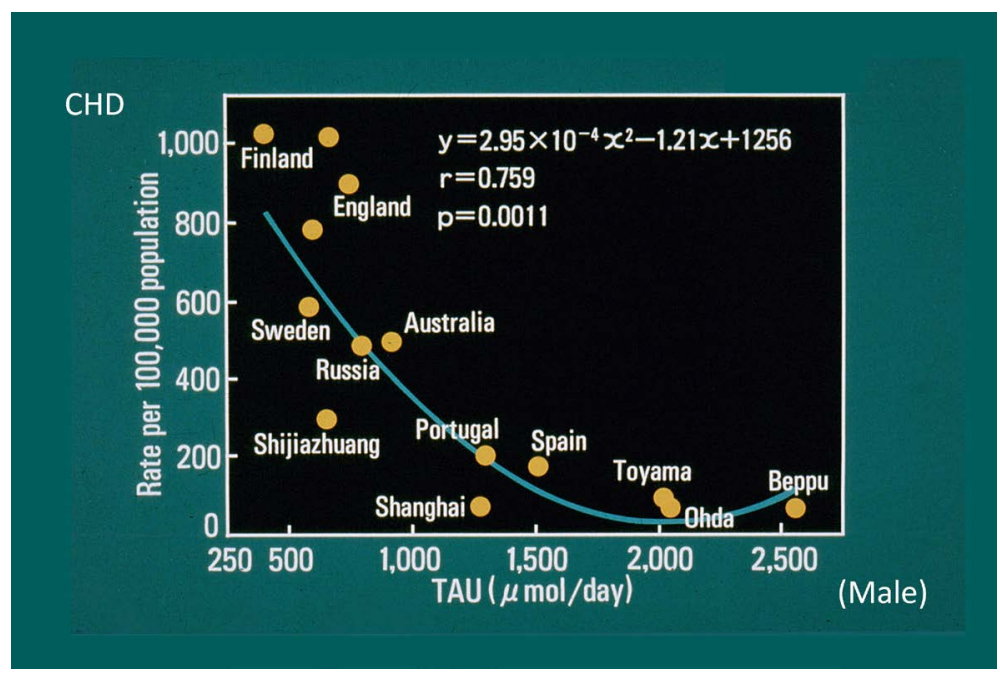

Figure 5. 24U taurine (TAU) and age-adjusted mortality rates of CHD. 


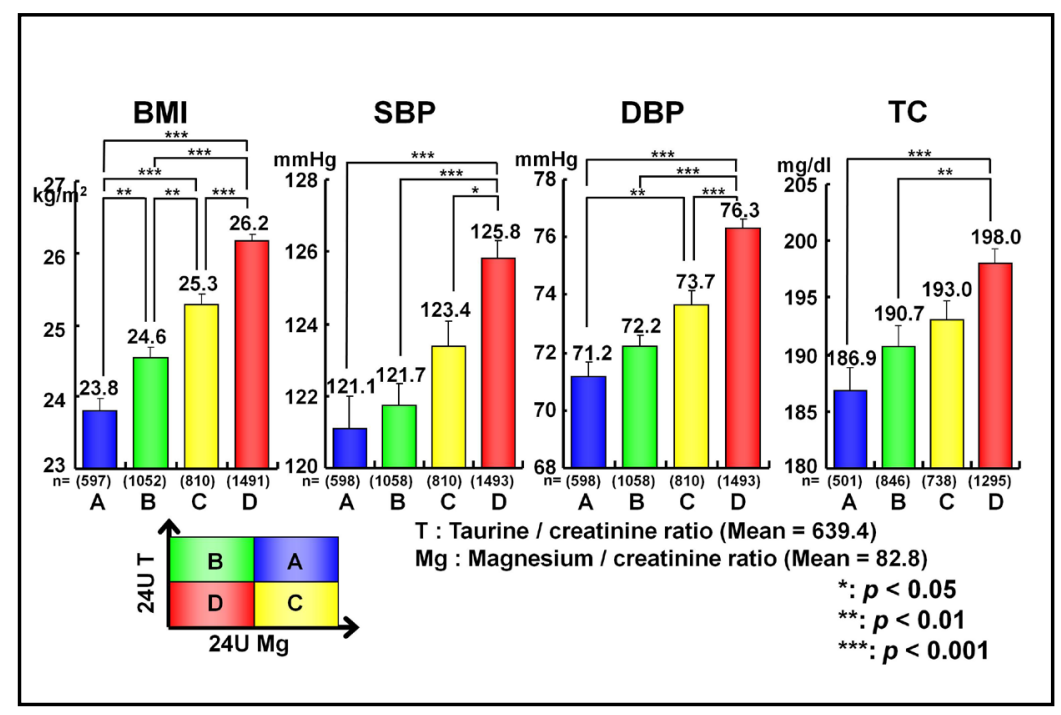

Figure 6. Cardiovascular risks associated with 24U Taurine (T) and Magnesium (Mg) excretions.

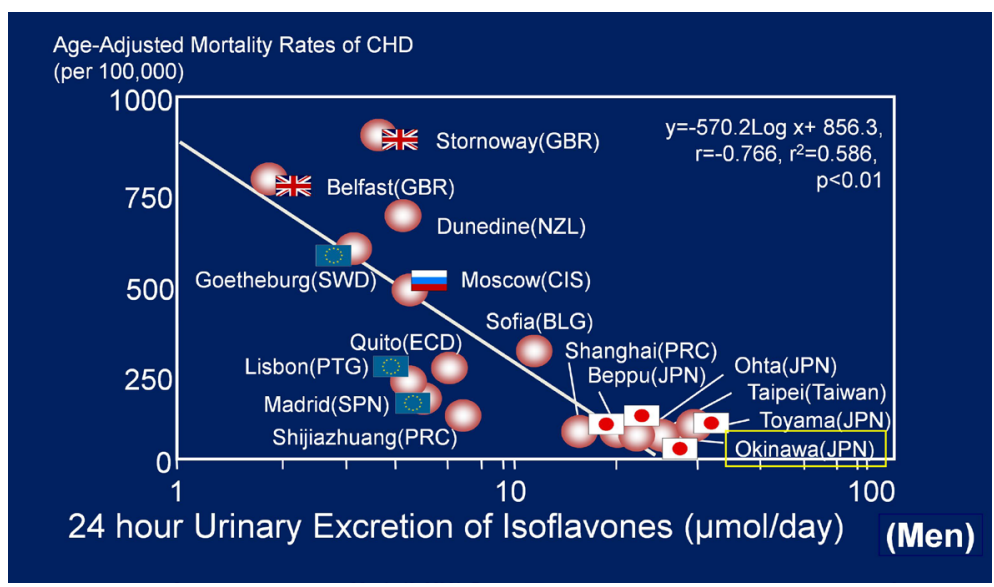

Figure 7. 24U isoflavones and age-adjusted mortality rates of CHD.

tervention studies have been carried out by utilizing 24U biomarkers. For example, Japanese immigrants from Okinawa, Japan are now living in Hawaii and Brazil, and the worldly known No. 1 longevity in Okinawans living in Japan was lost in Brazil.

Risks of lifestyle-related diseases such as obesity, hypertension and hypercholesterolemia were definitely increased in the immigrants in Hawaii and Brazil. The prevalence of hyperglycemia was particularly increased in Japanese immigrants in Brazil [10].

Nutritional biomarker analyses in $24 \mathrm{U}$ indicated soy isoflavone and $\mathrm{T}$ excretions were markedly decreased in Brazil compared with Okinawans living in Okinawa, indicating the marked reduction of soy and fish intakes in Brazil. Therefore, Okinawans living in Brazil were given soy breads containing $25 \mathrm{~g}$ soy protein a day for 4 to 8 weeks. BP and atherogenic index calculated as LDL to HDL ratios were significantly decreased as $24 \mathrm{U}$ isoflavone excretions were increased [10].

CARDIAC study showed Tibetans living in Lhasa at 3800 meters over the sea level had very high prevalence of hypertension because of their high salt intake proven by $24 \mathrm{U}$ collection. Their $24 \mathrm{U} \mathrm{T}$ excretion was lowest in the world because of their strict religious discipline not to eat fish at all. Therefore, Tibetans living in Namche Bazar, Nepal were given $3 \mathrm{~g}$ of $\mathrm{T}$ a day solved in tea for 2 months and their SBP and DBP were significantly decreased in 2 months by the intervention trial [10].

Since CARDIAC study indicates the ideal dietary intake of salt, vegetables, soybeans and fish by $24 \mathrm{U}$ bio- 
marker analyses, 2 kinds of lunch boxes were designed as "healthy lunch" containing low $\mathrm{Na}$ and $\mathrm{Na} / \mathrm{K}$ ratios and "fortified healthy lunch" containing soy and fish nutrients in addition of low $\mathrm{Na}$ and $\mathrm{Na} / \mathrm{K}$ ratios of healthy lunch, and both lunch boxes were given for 4 weeks to 2 randomized groups, each of 30 business men with relatively higher risks of metabolic syndrome [11]. Biomarker analyses showed both urinary $\mathrm{Na}$ and $\mathrm{Na} / \mathrm{K}$ ratio was significantly decreased in 4 weeks in both groups taking healthy and fortified healthy lunch boxes. BMI and DBP were significantly decreased in 4 weeks in accordance with changes in urinary biomarkers such as salt intake reduction from 14 to $11 \mathrm{~g}$ a day.

Since salt intake estimated by $24 \mathrm{U}$ Na excretion was significantly positively related with mortality rates of stroke in CARDIAC study, $3 \mathrm{~g}$ reduction of salt intake a day, if plotted on the simple regression line obtained by this worldwide study, would correspond to about $30 \%$ reduction of stroke mortality, if such lunch boxes could be taken life-long. Moreover, 24U isoflavone excretion, the biomarker of soy intakes increased markedly in the fortified healthy lunch group. Correspondingly, LDL levels decreased and HDL increased significantly only in the fortified healthy lunch group. Therefore, atherogenic index, the ratio of LDL to HDL, decreased significantly $11 \%$, from the baseline level only in the fortified healthy lunch group, indicating soy isoflavone intake may contribute to the prevention of atherosclerosis.

Australian aboriginals had the highest risks of lifestyle-related diseases such as obesity, hypertension and diabetes among CARDIAC study populations examined in the world [12]. The prevalence of hypertension was over $40 \%$ at the age of 30, higher than the age-matched Japanese and Australians. We offered them soy bean breads containing $25 \mathrm{~g}$ of soy protein a day and wheat breads as the placebo for 8 weeks in a randomized placebo controlled intervention trial. Soy bread group showed a significant reduction in DBP and atherogenic index in comparison with wheat bread group, indicating $25 \mathrm{~g}$ of soy protein with over $100 \mathrm{mg}$ of isoflavones a day could reduce CVD risks, if continued long enough (Figure 8) [12].

\section{24U Biomarkers for Population Approach to Hypertension}

24U biomarkers are not only useful for intervention trial but also for population strategy for cardiovascular health promotion. A population strategy by 10 -year campaign to eat rice and more soy beans with traditional Japanese food and to reduce salt intake was carried out in Hyogo Prefecture in the center of Japan, 5.6 million population of which corresponded to one 20th of Japanese population (Figure 9) [13]. The results were monitored in 9 towns by checking 24U biomarkers of nutrition.

The results of $24 \mathrm{U}$ analysis demonstrated the frequency distribution of 24-hour salt excretion in 2002 shifted to the left, toward the lower level, in 2004 (Figure 10). The average of 24U salt excretion was reduced in 2004 down to $10.1 \mathrm{~g}$, by $1.9 \mathrm{~g}$ reduction, significantly from the mean in $2002,12 \mathrm{~g}$ a day.

The ratio of the inhabitants whose daily salt intakes were less than $10 \mathrm{~g}$, the goal of daily salt intakes settled at that time by Japanese government, was significantly increased from $36 \%$, up to $53 \%$, over a half of the population in 2004. Correspondingly, the percentage of the people with SBP less than $140 \mathrm{mmHg}, 68 \%$ in 2002 was significantly increased up to $76 \%$ in 2004 (Figure 11). The percentage of people with SBP range equal to or over 140 and below 160, 23\% in 2002, was significantly decreased down to $17 \%$ in 2004 . The salt reduction campaign proven by $24 \mathrm{U}$ analysis was effective for decreasing the SBP range of borderline and mild hypertensives down to the range of normotensives.

In response to the campaign to eat more soy beans, the average of isoflavone intakes estimated by $24 \mathrm{U}$ isoflavone excretion increased yearly up to 1.7 times greater in 2004 than the levels in 2002. The interaction of soy intake with salt-induced BP rise was analyzed further, and among the population whose isoflavone intakes were less than $25 \mathrm{mg}$ a day, significant differences were noted both in SBP and DBP between the people taking equal to, or over $10 \mathrm{~g}$ of salt a day and those taking less salt than $10 \mathrm{~g}$ a day. In contrast, among the population whose isoflavone intakes were more than $25 \mathrm{mg}$ a day, people taking equal to, or over $10 \mathrm{~g}$ of salt a day did not show any differences from the people taking less salt daily. Since CARDIAC study indicated the positive relationships of SBP and DBP with salt intake were not significant before menopause, enough isoflavone intake may attenuate salt-induced BP rise because of its estrogenic activity [14].

Since our worldwide health survey indicated the stroke mortality was positively associated with $24 \mathrm{U}$ salt excretion, and CHD mortality was inversely associated with urinary isoflavone excretion, 1.9 g reduction of 24U $\mathrm{NaCl}$ excretion and 1.7 times increases in urinary isoflavone excretion, if maintained continuously further, are expected to reduce stroke mortality and CHD mortality greatly down to Okinawan's lowest mortality in Japan. 


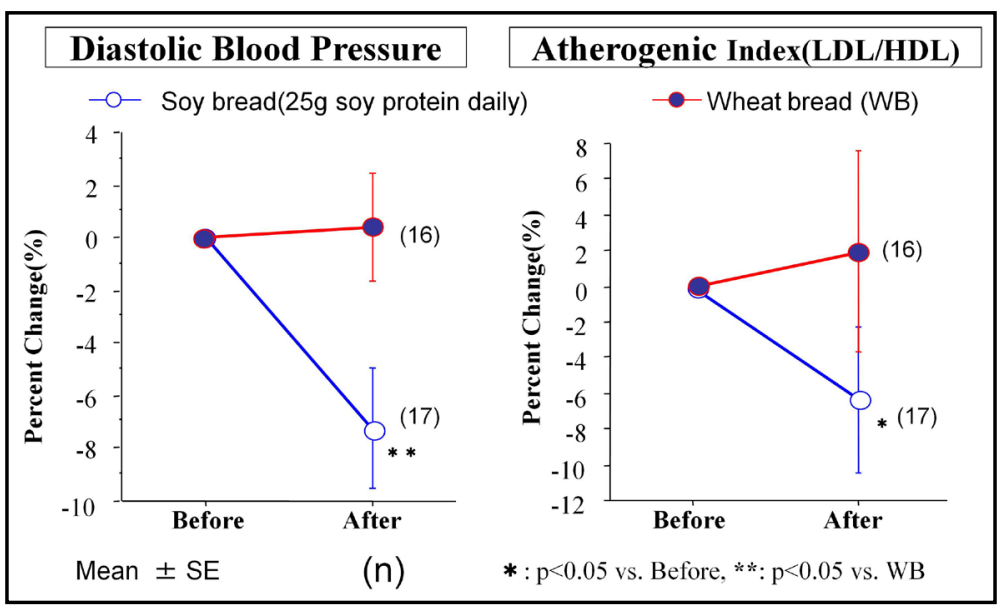

Figure 8. Soy or wheat bread effect in Australian aboriginals-an 8-week randomized placebo-controlled intervention study.

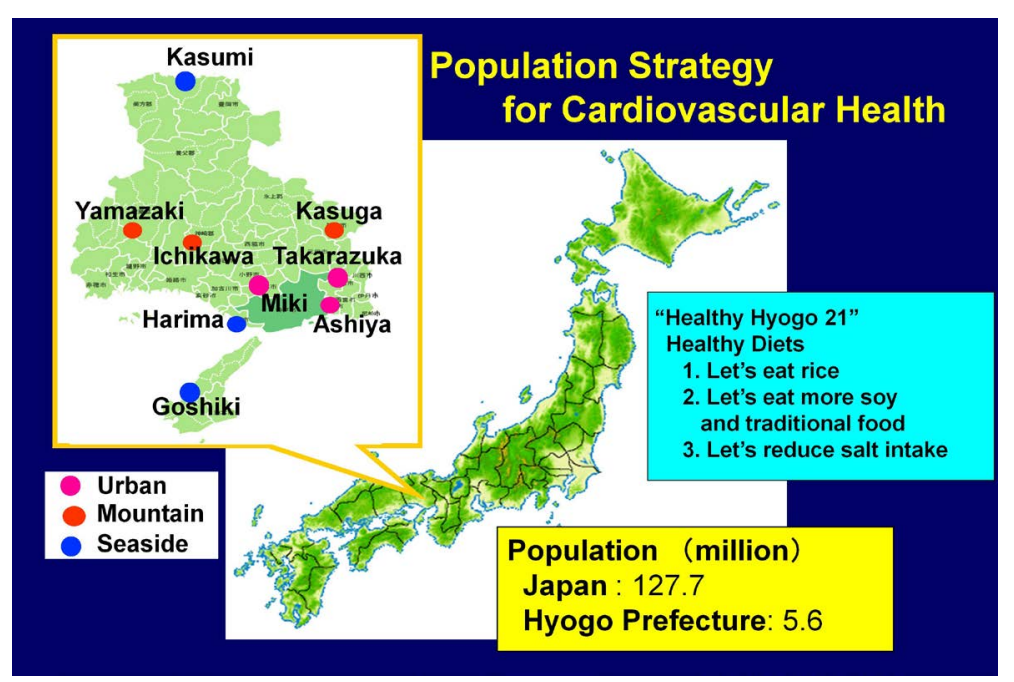

Figure 9. Population approach to hypertension-related diseases in Hyogo Prefecture.

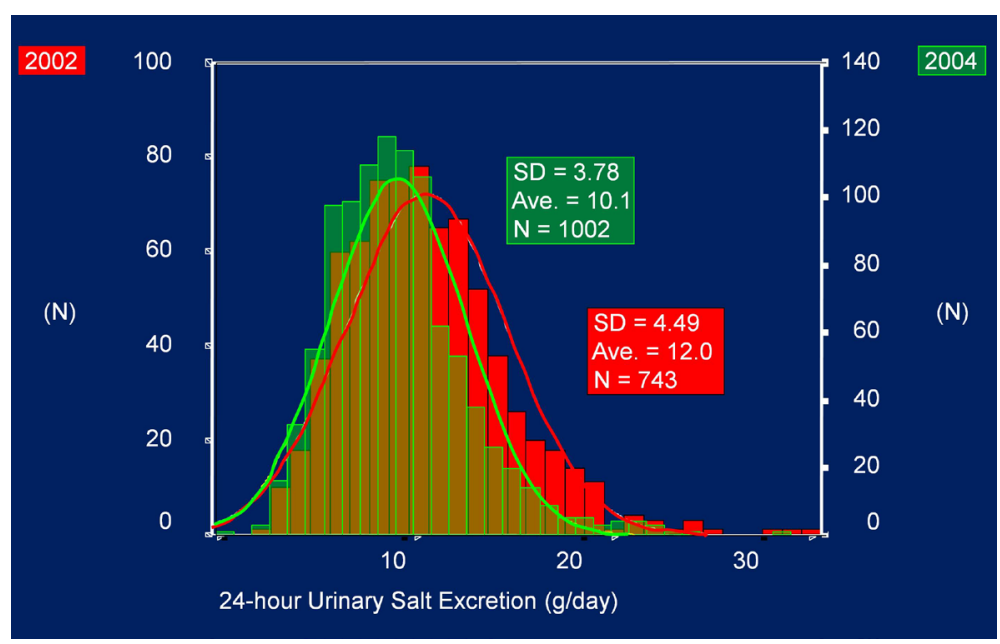

Figure 10. Distribution of 24U salt excretion in 2002 and 2004. 


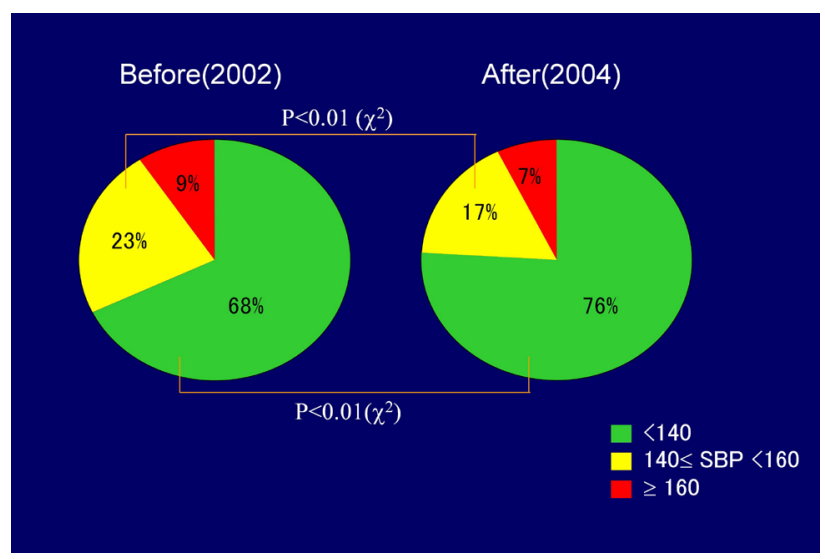

Figure 11. Significant reduction of mild hypertensives after health promotion campaign.

Therefore, 24U biomarker analyses are useful for the evaluation of population strategy, and would contribute to health promotion based on the objective data of nutritional improvement.

\section{Conclusion}

A simple method for $24 \mathrm{U}$ collection has enabled us to utilize nutritional biomarkers for hypertension-related disease risk analyses, and to apply the objective nutritional evaluation to intervention studies as well as to population strategy for nutritional improvement, and therefore, the methodology will hopefully contribute to health promotion and disease prevention in the world.

\section{Acknowledgements}

The authors are grateful for the contribution of many investigators and volunteers in 61 study sites in the world to WHO-CARDIAC study, which was supported by grants-in-aid from Japanese Government and the donation to WHO from about 300,000 individuals and companies such as Otsuka Pharmaceutical Co., Ltd. Tokyo, as well as for the great cooperation of Victorian Aboriginal Health Service, Melbourne, Australia and Hyogo Prefecture Health Promotion Association, Kobe, Japan.

\section{References}

[1] Yamori, Y. (1984) The Stroke-Prone Spontaneously Hypertensive Rat: Contribution to Risk Factor Analysis and Prevention of Hypertensive Diseases. In: de Yong, W., Ed., Handbook of Hypertension Vol. 4: Experimental and Genetic Models of Hypertension, Elsevier, Amsterdam, 240-255.

[2] Yamori, Y., Nara, Y., Kihara, M., Mano, M. and Horie, R. (1984) Simple Method for Sampling Consecutive 24-Hour Urine for Epidemiological and Clinical Studies. Clinical and Experimental Hypertension, A6, 1160-1967.

[3] Yamori, Y. and Lenfent, C. (1987) Prevention of Cardiovascular Diseases: An Approach to Active Long Life. Elsevier, Amsterdam.

[4] Yamori, Y., Nara, Y., Mizushima, S., Mano, M., Sawamura, M., Kihara, M., Horie, R. and Hatano, S. (1990) International Cooperative Study on the Relationship between Dietary Factors and Blood Pressure: A Report from the Cardiovascular Diseases and Alimentary Comparison Study. Journal of Cardiovascular Pharmacology, 16, S43-S47. http://dx.doi.org/10.1097/00005344-199000168-00018

[5] Yamori, Y., Liu, L., Mizushima, S., Ikeda, K. and Nara, Y. (2006) Male Cardiovascular Mortality and Dietary Markers in 25 Population Samples of 16 Countries. Journal of Hypertension, 24, 1499-1505. http://dx.doi.org/10.1097/01.hjh.0000239284.12691.2e

[6] Yamori, Y. (2006) Food Factors for Atherosclerosis Prevention: Asian Perspective Derived from Analyses of Worldwide Dietary Biomarkers. Experimental \& Clinical Cardiology, 11, 94-98.

[7] Yamori, Y., Mizushima, S., Sawamura, M. and Nara, Y. (1994) Nutritional Factors for Hypertension and Major Cardiovascular Diseases: International Cooperative Studies for Dietary Prevention (in Japanese). Deutsche Medizinische Wochenschrift, 15, 1825-1841. 
[8] Yamori, Y., Liu, L., Mori, M., Sagara, M., Murakami, S., Nara, Y. and Mizushima, S. (2009) Taurine as the Nutritional Factor for the Longevity of the Japanese Revealed by a Worldwide Epidemiological Survey. Advances in Experimental Medicine and Biology, 643, 13-25. http://dx.doi.org/10.1007/978-0-387-75681-3_2

[9] Yamori, Y., Taguchi, T., Mori, H. and Mori, M. (2010) Low Cardiovascular Risks in the Middle Aged Males and Females Excreting Greater 24U Taurine and Magnesium in 41 WHO-CARDIAC Study Populations in the World. Journal of Biomedical Science, 17, S21. http://dx.doi.org/10.1186/1423-0127-17-S1-S21

[10] Yamori, Y. (2006) Soy for "Health for All”: Message from WHO-CARDIAC Study and Dietary Intervention Studies. In: Sugano, M., Ed., Soy in Health and Diseases Prevention, Taylor \& Francis, Florida, 107-121.

[11] Mori, H., Mori, M. and Yamori, Y. (2009) Preventive Food for Risk Reduction of Lifestyle-Related Diseases: The Benefit Proven by “A Well-Balanced Meal a Day Project”. Proceedings of the International Symposium: Food Culture: Development and Education/Building Food Culture and Education for Longevity, 20-Year CARDIAC Study Symposium Supported by UNESCO, Nonprofit Organization World Health Frontier Institute, 105-109.

[12] Mori, M. and Thorpe, M. (2009) Australian Aboriginals with Highest Risks and a New Attempt for Their Nutritional Improvement. Proceedings of the International Symposium: Food Culture: Development and Education/Building Food Culture and Education for Longevity, 20-Year CARDIAC Study Symposium Supported by UNESCO, Nonprofit Organization World Health Frontier Institute, 97-101.

[13] Mori, M., Sagara, M., Ohnishi, C., Imai, K. and Yamori, Y. (2007) Effect of Population Strategy on Hypertension (in Japanese). Therapeutic Research, 28, 1788-1791.

[14] Yamori, Y., Liu, L., Ikeda, K., Mizushima, S., Nara, Y. and Simpson, O. (2001) Different Associations of Blood Pressure with 24-Hour Urinary Sodium Excretion among Pre- and Post-Menopausal Women. Journal of Hypertension, 19, 535-538. http://dx.doi.org/10.1097/00004872-200103001-00003 